\title{
Connecting Stalk
}

National Cancer Institute

\section{Source}

National Cancer Institute. Connecting Stalk. NCI Thesaurus. Code C34132.

Extraembryonic mesoderm that links the embryonic disc to the chorionic membrane wall, and eventually fuses with the yolk sac stalk to form the umbilical cord. 